\title{
The Simplified Tikhonov Regularization Method for Identifying the Unknown Source for the Modified Helmholtz Equation
}

\author{
Fan Yang, ${ }^{1}$ HengZhen Guo, ${ }^{2}$ and XiaoXiao $\mathrm{Li}^{1}$ \\ ${ }^{1}$ School of Science, Lanzhou University of Technology, Lanzhou, Gansu 730050, China \\ ${ }^{2}$ Institute of Education, Lanzhou City University, Lanzhou, Gansu 730070, China \\ Correspondence should be addressed to Fan Yang, yfggd114@163.com
}

Received 20 December 2010; Revised 6 April 2011; Accepted 25 April 2011

Academic Editor: Paulo Batista Gonçalves

Copyright $@ 2011$ Fan Yang et al. This is an open access article distributed under the Creative Commons Attribution License, which permits unrestricted use, distribution, and reproduction in any medium, provided the original work is properly cited.

This paper discusses the problem of determining an unknown source which depends only on one variable for the modified Helmholtz equation. This problem is ill-posed in the sense that the solution (if it exists) does not depend continuously on the data. The regularization solution is obtained by the simplified Tikhonov regularization method. Convergence estimate is presented between the exact solution and the regularization solution. Moreover, numerical results are presented to illustrate the accuracy and efficiency of this method.

\section{Introduction}

Inverse source problems arise in many branches of science and engineering, for example, heat conduction, crack identification, electromagnetic theory, geophysical prospecting, and pollutant detection. For the heat source identification, there has been a large number of research results for different forms of heat source [1-6]. To the author's knowledge, there were few papers for identifying the unknown source on the modified Helmholtz equation which is pointed out in [7] by regularization method.

In this paper, we consider the following inverse problem: to find a pair of functions $(u(x, y), f(x))$ satisfying

$$
\begin{gathered}
\Delta u(x, y)-k^{2} u(x, y)=f(x), \quad 0<x<\pi, 0<y<+\infty, \\
u(0, y)=u(\pi, y)=0, \quad 0 \leq y<+\infty,
\end{gathered}
$$




$$
\begin{gathered}
u(x, 0)=0, \quad 0 \leq x \leq \pi, \\
\left.u(x, y)\right|_{y \rightarrow \infty} \text { bounded, } \quad 0 \leq x \leq \pi, \\
u(x, 1)=g(x), \quad 0 \leq x \leq \pi,
\end{gathered}
$$

where $f(x)$ is the unknown source depending only on one spatial variable, $u(x, 1)=g(x)$ is the supplementary condition, and the constant $k>0$ is the wave number. In applications, input data $g(x)$ can only be measured, and there will be measured data function $g^{\mathcal{S}}(x)$ which is merely in $L^{2}(0, \pi)$ and satisfies

$$
\left\|g-g^{\delta}\right\|_{L^{2}(0, \pi)} \leq \delta
$$

where the constant $\delta>0$ represents a noise level of input data.

It is easy to derive a solution of problem (1.1) by the method of separation of variables

$$
u(x, y)=-\sum_{n=1}^{\infty} \frac{1-e^{-\sqrt{n^{2}+k^{2}} y}}{n^{2}+k^{2}} f_{n} X_{n}
$$

where

$$
\left\{X_{n}=\sqrt{\frac{2}{\pi}} \sin n x,(n=1,2 \ldots)\right\}
$$

is an orthogonal basis in $L^{2}(0, \pi)$, and

$$
f_{n}=\sqrt{\frac{2}{\pi}} \int_{0}^{\pi} f(x) \sin n x d x .
$$

By the supplementary condition, we define the operator $K: f \rightarrow g$, then we have

$$
g(x)=K f(x)=-\sum_{n=1}^{\infty} \frac{1-e^{-\sqrt{n^{2}+k^{2}}}}{n^{2}+k^{2}} f_{n} X_{n} .
$$

It is easy to see that $K$ is a linear compact operator and the singular values $\left\{\sigma_{n}\right\}_{n=1}^{n=\infty}$ of $K$ satisfy

$$
\begin{aligned}
& \sigma_{n}=-\frac{1-e^{-\sqrt{n^{2}+k^{2}}}}{n^{2}+k^{2}}, \\
& g_{n}=-\frac{1-e^{-\sqrt{n^{2}+k^{2}}}}{n^{2}+k^{2}} f_{n}\left(X_{n}, X_{n}\right),
\end{aligned}
$$

that is,

$$
f_{n}=\sigma_{n}^{-1} g_{n}
$$


where

$$
g_{n}=\sqrt{\frac{2}{\pi}} \int_{0}^{\pi} g(x) \sin n x d x .
$$

Therefore,

$$
f(x)=K^{-1} g(x)=\sum_{n=1}^{\infty} \frac{1}{\sigma_{n}}\left(g, X_{n}\right) X_{n}=-\sum_{n=1}^{\infty} \frac{n^{2}+k^{2}}{1-e^{-\sqrt{n^{2}+k^{2}}}} g_{n} X_{n}
$$

Note that $1 / \sigma_{n}=O\left(n^{2}\right)$ as $n \rightarrow \infty$, thus the exact data function $g(x)$ must satisfy the property that $\left(g, X_{n}\right)$ decays rapidly as $O\left(n^{-2}\right)$. As for the measured data function $g^{\delta}(x)$ is only in $L^{2}(0, \pi)$, we cannot expect the coefficient $g_{n}^{\delta}$ of $g^{\delta}(x)$ has the same decay rate. Thus, the problem (1.1) is ill posed. It is impossible to gain the unknown source using classical methods. In the following sections, we will use the simplified Tikhonov method to deal with the ill posed problem. Before doing that, we impose an a priori bound on the unknown source; that is,

$$
\|f(\cdot)\|_{H^{p}(0, \pi)} \leq E, \quad p>0
$$

where $E>0$ is a constant and $\|\cdot\|_{H^{p}(0, \pi)}$ denotes the norm in Sobolev space which is defined by [8] as follows:

$$
\|f(\cdot)\|_{H^{p}(0, \pi)}=\left(\sum_{n=1}^{\infty}\left(1+n^{2}\right)^{p}\left|\left(f, X_{n}\right)\right|^{2}\right)^{1 / 2} .
$$

The simplified Tikhonov regularization method was based on the Tikhonov regularization method. Skillfully simplifying the filter gained by the Tikhonov regularization, a better regularization approximation solution of the inverse problem was obtained. This idea initially came from Carasso, the author who modified the filter gained by the Tikhonov regularization method and obtained the order optimal error estimate in [9]. By this method, $\mathrm{Fu}[10]$ considered the inverse heat conduction problem on a general sideways parabolic equation, and Cheng et al. [11,12] considered the spherically symmetric inverse problem.

This paper is organized as follows. Section 2 gives some auxiliary results. Section 3 gives a simplified Tikhonov regularization solution and error estimation. Section 4 gives two examples to illustrate the accuracy and efficiency of this methods. Section 5 puts an end to this paper with a brief conclusion.

\section{Some Auxiliary Results}

Now, we give some important Lemmas, which are very useful for our main conclusion.

Lemma 2.1. For $n \geq 1$ and $k$ is a positive constant, there holds

$$
\frac{1}{1-e^{-\sqrt{n^{2}+k^{2}}}} \leq 2
$$


Lemma 2.2. For $0<\alpha<1$, there holds the following inequalities:

$$
\begin{gathered}
\sup _{n \geq 1}\left(1-\frac{1}{1+\alpha^{2} n^{4}}\right)\left(1+n^{2}\right)^{-p / 2} \leq \max \left\{\alpha^{2}, \alpha^{p / 2}\right\}, \\
\sup _{n \geq 1} \frac{n^{2}+k^{2}}{\left(1-e^{-\sqrt{n^{2}+k^{2}}}\right)\left(1+\alpha^{2} n^{4}\right)} \leq \frac{2}{\alpha}+2 k^{2} .
\end{gathered}
$$

Proof. Let

$$
G(n):=\left(1-\frac{1}{1+\alpha^{2} n^{4}}\right)\left(1+n^{2}\right)^{-p / 2} .
$$

The proof of (2.2) can be separated from two cases.

Case 1. For large values of $n$, that is, $n \geq n_{0}:=1 / \sqrt{\alpha}$, we get

$$
G(n) \leq\left(1+n^{2}\right)^{-p / 2} \leq n^{-p} \leq n_{0}^{-p}=\alpha^{p / 2}
$$

Case 2. $1 \leq n<n_{0}$, we obtain

$$
G(n)=\frac{\alpha^{2} n^{4}}{1+\alpha^{2} n^{4}}\left(1+n^{2}\right)^{-p / 2} \leq \alpha^{2} n^{4}\left(1+n^{2}\right)^{-p / 2} \leq \alpha^{2} n^{4-p} .
$$

If $0<p \leq 4$, above inequality becomes into

$$
G(n) \leq \alpha^{2} n^{4-p}<\alpha^{2} n_{0}^{4-p}=\alpha^{p / 2} .
$$

If $p>4$, we get

$$
G(n) \leq \alpha^{2} n^{4-p} \leq \alpha^{2}
$$

Combining (2.4) with (2.6) and (2.7), the first inequality equation is obtained.Let

$$
\begin{aligned}
B(n) & :=\frac{n^{2}+k^{2}}{\left(1-e^{-\sqrt{n^{2}+k^{2}}}\right)\left(1+\alpha^{2} n^{4}\right)} \\
& =\frac{n^{2}}{\left(1-e^{-\sqrt{n^{2}+k^{2}}}\right)\left(1+\alpha^{2} n^{4}\right)}+\frac{k^{2}}{\left(1-e^{-\sqrt{n^{2}+k^{2}}}\right)\left(1+\alpha^{2} n^{4}\right)} \\
& :=H(n)+J(n) .
\end{aligned}
$$


Using Lemma 1, we obtain

$$
H(n) \leq \frac{2 n^{2}}{1+\alpha^{2} n^{4}}
$$

Let

$$
L(n):=\frac{2 n^{2}}{1+\alpha^{2} n^{4}}
$$

then

$$
L^{\prime}(n)=\frac{4 n\left(1-\alpha^{2} n^{4}\right)}{\left(1+\alpha^{2} n^{4}\right)^{2}}
$$

Setting $L^{\prime}(n)=0$, we can obtain $n_{1}=1 / \sqrt{\alpha}$. It is easy to see that $n_{1}=1 / \sqrt{\alpha}$ is a unique maximal value point of $L(n)$.

So,

$$
\begin{aligned}
& L(n) \leq \frac{2 n_{1}^{2}}{1+\alpha^{2} n_{1}^{2}} \leq 2 n_{1}^{2}=\frac{2}{\alpha^{\prime}} \\
& J(n) \leq \frac{k^{2}}{1-e^{-\sqrt{n^{2}+k^{2}}}} \leq 2 k^{2} .
\end{aligned}
$$

So, we get

$$
B(n) \leq \frac{2}{\alpha}+2 k^{2}
$$

This completes the proof.

\section{A Simplified Tikhonov Regularization Method}

Since problem (1.1) is an ill-posed problem, we give an approximate solution of $f(x)$ by a Tikhonov regularization method which minimizes the quantity

$$
\left\|K f^{\delta}-g^{\delta}\right\|^{2}+\alpha^{2}\left\|f^{\delta}\right\|^{2}
$$

Then, by Theorem 2.12 in [8], the unique solution of the minimization problem (3.1) is equal to solve the following normal equation:

$$
K^{*} K f^{\mathcal{\delta}}(x)+\alpha^{2} f^{\delta}(x)=K^{*} g^{\delta}(x)
$$

that is,

$$
f^{\mathcal{S}}(x)=\left[K^{*} K+\alpha^{2} I\right]^{-1} K^{*} g^{\delta}(x)
$$


Because $K$ is a linear self-adjoint compact operator, that is, $K^{*}=K$, we have the equivalent form

$$
f^{\mathcal{\delta}}(x)=\left[K^{2}+\alpha^{2} I\right]^{-1} K g^{\delta}(x)
$$

We define function of a compact self-adjoint operator $K$ by the spectral mapping theorem in the following way.

Definition 3.1 (see[13]). If $f(x)$ is a real-valued continuous function on the spectrum $\sigma(K)$, we define $f(K)$ by

$$
f(K) x=\sum_{n} f\left(\lambda_{n}\right)\left(x, \omega_{n}\right) \omega_{n}
$$

where $K$ is a compact self-adjoint, $\lambda_{n} \in \sigma(K)$, and $\omega_{n}$ are the corresponding orthogonal eigenvectors.

So, we obtain

$$
\begin{aligned}
f^{\delta}(x) & =-\sum_{n=1}^{\infty} \frac{\left(1-e^{-\sqrt{n^{2}+k^{2}}}\right) /\left(n^{2}+k^{2}\right)}{\alpha^{2}+\left(\left(1-e^{-\sqrt{n^{2}+k^{2}}}\right) /\left(n^{2}+k^{2}\right)\right)^{2}}\left(g^{\delta}(x), X_{n}\right) X_{n} \\
& =-\sum_{n=1}^{\infty} \frac{\left(n^{2}+k^{2}\right) /\left(1-e^{-\sqrt{n^{2}+k^{2}}}\right)}{1+\alpha^{2}\left(\left(n^{2}+k^{2}\right) /\left(1-e^{-\sqrt{n^{2}+k^{2}}}\right)\right)^{2}}\left(g^{\delta}(x), X_{n}\right) X_{n} \\
& =-\sum_{n=1}^{\infty} \frac{\left(n^{2}+k^{2}\right) /\left(1-e^{-\sqrt{n^{2}+k^{2}}}\right)}{1+\alpha^{2}\left(\left(n^{2}+k^{2}\right) /\left(1-e^{-\sqrt{n^{2}+k^{2}}}\right)\right)^{2}} g_{n}^{\delta} X_{n} .
\end{aligned}
$$

Comparing (1.10) with (3.6), we can find that the procedure consists in replacing the unknown $g(x)$ with an appropriately filtered noised data $g^{\delta}(x)$. The filter in (3.6) attenuates the coefficient $g_{n}^{\delta}$ of $g^{\delta}(x)$ in a manner consistent with the goal of minimizing quantity (3.1). By this idea, we can use a much better filter $1 /\left(1+\alpha^{2} n^{4}\right)$ to replace the filter $1 /\left(1+\alpha^{2}\left(\left(n^{2}+k^{2}\right) /\left(1-e^{-\sqrt{n^{2}+k^{2}}}\right)\right)^{2}\right)$ and give another approximation $f_{\alpha}^{\mathcal{S}}(x)$ of the solution $f(x)$.

We define a regularization approximate solution of problem (1.1) for noisy data $g^{\delta}(x)$ which is called the simplified Tikhonov regularized solution of problem (1.1) as follows:

$$
f_{\alpha}^{\delta}(x):=-\sum_{n=1}^{\infty} \frac{n^{2}+k^{2}}{\left(1-e^{-\sqrt{n^{2}+k^{2}}}\right)\left(1+\alpha^{2} n^{4}\right)}\left(g^{\delta}, X_{n}\right) X_{n}
$$


Theorem 3.2. Let $f_{\alpha}^{\delta}(x)$ be the simplified Tikhonov approximation of the solution $f(x)$ of problem (1.1). Let $g^{\delta}(x)$ be measured data at $y=1$ satisfying (1.2), and let priori condition (1.11) hold for $p>0$. If one selects

$$
\alpha=\left(\frac{\delta}{E}\right)^{2 /(p+2)}
$$

then the following estimate holds:

$$
\left\|f(\cdot)-f_{\alpha}^{\mathcal{\delta}}(\cdot)\right\|_{L^{2}(0, \pi)} \leq 2 \delta^{p /(p+2)} E^{2 /(p+2)}\left(1+\frac{1}{2} \max \left\{1,\left(\frac{\delta}{E}\right)^{(4-p) /(p+2)}\right\}\right)+2 k^{2} \delta .
$$

Proof. Due to the triangle inequality, we have

$$
\begin{aligned}
& \left\|f-f_{\alpha}^{\delta}\right\|_{L^{2}(0, \pi)} \\
& =-\sum_{n=1}^{\infty} \frac{n^{2}+k^{2}}{1-e^{-\sqrt{n^{2}+k^{2}}}}\left(g, X_{n}\right) X_{n} \\
& -\left(-\sum_{n=1}^{\infty} \frac{n^{2}+k^{2}}{\left(1+\alpha^{2} n^{4}\right)\left(1-e^{-\sqrt{n^{2}+k^{2}}}\right)}\left(g^{\delta}, X_{n}\right) X_{n}\right) \|_{L^{2}(0, \pi)} \\
& =\| \sum_{n=1}^{\infty} \frac{n^{2}+k^{2}}{1-e^{-\sqrt{n^{2}+k^{2}}}}\left(g, X_{n}\right) X_{n} \\
& -\sum_{n=1}^{\infty} \frac{n^{2}+k^{2}}{\left(1+\alpha^{2} n^{4}\right)\left(1-e^{-\sqrt{n^{2}+k^{2}}}\right)}\left(g^{\delta}, X_{n}\right) X_{n} \|_{L^{2}(0, \pi)} \\
& \leq\left\|\sum_{n=1}^{\infty} \frac{n^{2}+k^{2}}{1-e^{-\sqrt{n^{2}+k^{2}}}}\left(g, X_{n}\right) X_{n}-\sum_{n=1}^{\infty} \frac{n^{2}+k^{2}}{\left(1+\alpha^{2} n^{4}\right)\left(1-e^{-\sqrt{n^{2}+k^{2}}}\right)}\left(g, X_{n}\right) X_{n}\right\|_{L^{2}(0, \pi)} \\
& +\| \sum_{n=1}^{\infty} \frac{n^{2}+k^{2}}{\left(1+\alpha^{2} n^{4}\right)\left(1-e^{-\sqrt{n^{2}+k^{2}}}\right)}\left(g, X_{n}\right) X_{n} \\
& -\sum_{n=1}^{\infty} \frac{n^{2}+k^{2}}{\left(1+\alpha^{2} n^{4}\right)\left(1-e^{-\sqrt{n^{2}+k^{2}}}\right)}\left(g^{\delta}, X_{n}\right) X_{n} \|_{L^{2}(0, \pi)} \\
& =\left\|\sum_{n=1}^{\infty} \frac{n^{2}+k^{2}}{1-e^{-\sqrt{n^{2}+k^{2}}}}\left(g, X_{n}\right) X_{n}\left(1-\frac{1}{1+\alpha^{2} n^{4}}\right)_{L^{2}(0, \pi)}\right\|
\end{aligned}
$$




$$
\begin{aligned}
&+\left\|\sum_{n=1}^{\infty} \frac{n^{2}+k^{2}}{\left(1+\alpha^{2} n^{4}\right)\left(1-e^{-\sqrt{n^{2}+k^{2}}}\right)}\left(g-g^{\delta}, X_{n}\right) X_{n}\right\|_{L^{2}(0, \pi)} \\
& \leq\left.\left\|\sum_{n=1}^{\infty}\left(1-\frac{1}{1+\alpha^{2} n^{4}}\right)\left(f, X_{n}\right) X_{n}\left(1+n^{2}\right)^{p / 2}\left(1+n^{2}\right)^{-p / 2}\right\|_{L^{2}(0, \pi)}\right)\left\|\sum_{n=1}^{\infty}\left(g-g^{\delta}, X_{n}\right) X_{n}\right\|_{L^{2}(0, \pi)} \\
&+\sup _{n \geq 1}\left(\frac{n^{2}+k^{2}}{\left(1+\alpha^{2} n^{4}\right)\left(1-e^{-\sqrt{n^{2}+k^{2}}}\right)}\right)\left\|\left(f, X_{n}\right) X_{n}\left(1+n^{2}\right)^{p / 2}\right\|_{L^{2}(0, \pi)} \\
& \leq \sup _{n \geq 1}\left(\left(1-\frac{1}{1+\alpha^{2} n^{4}}\right)\left(1+n^{2}\right)^{-p / 2}\right)\left\|\sum_{n=1}^{\infty}\left(g-g^{\delta}, X_{n}\right) X_{n}\right\|_{L^{2}(0, \pi)} \\
&+\sup _{n \geq 1}\left(\frac{n^{2}+k^{2}}{\left(1+\alpha^{2} n^{4}\right)\left(1-e^{-\sqrt{n^{2}+k^{2}}}\right)}\right) \\
& \leq\left.\sup _{n \geq 1}\left(\left(1-\frac{1}{1+\alpha^{2} n^{4}}\right)\left(1+n^{2}\right)^{-p / 2}\right) E+\sup _{n \geq 1} \frac{n^{2}+k^{2}}{\left(1+\alpha^{2} n^{4}\right)\left(1-e^{-\sqrt{n^{2}+k^{2}}}\right)}\right) \delta \\
& \leq \max \left\{\alpha^{2}, \alpha^{(p / 2)}\right\} E+\frac{2}{\alpha} \delta+2 k^{2} \delta \\
&= \max \left\{\left(\frac{\delta}{E}\right)^{4 /(p+2)},\left(\frac{\delta}{E}\right)^{p / p+2}\right\} E+2\left(\frac{\delta}{E}\right)^{-2 /(p+2)} \delta+2 k^{2} \delta \\
& E^{2 /(p+2)}\left(1+\frac{1}{2} \max \left\{1,\left(\frac{\delta}{E}\right)^{4-p /(p+2)}\right\}\right)+2 k^{2} \delta .
\end{aligned}
$$

The proof is complete.

Remark 3.3. If $0<P \leq 4$,

$$
\left\|f(\cdot)-f_{\alpha}^{\delta}(\cdot)\right\|_{L^{2}(0, \pi)} \leq 3 \delta^{p /(p+2)} E^{2 /(p+2)}+2 k^{2} \delta \longrightarrow 0, \quad \text { as } \delta \longrightarrow 0 .
$$

If $p>4$,

$$
\left\|f(\cdot)-f_{\alpha}^{\delta}(\cdot)\right\|_{L^{2}(0, \pi)} \leq 2 \delta^{p /(p+2)} E^{2 /(p+2)}+\delta^{4 /(p+2)} E^{(p-2) /(p+2)}+2 k^{2} \delta \longrightarrow 0, \quad \text { as } \delta \longrightarrow 0 .
$$

Hence, $f_{\alpha}^{\delta}(x)$ can be viewed as the approximation of the exact solution $f(x)$. 
Table 1: Relative errors $\operatorname{rerr}(f)$ with $\varepsilon=0.01, k=2, N=10$ and $p=2$ for different $M$.

\begin{tabular}{lcccccccc}
\hline$M$ & 10 & 50 & 100 & 200 & 400 & 800 & 1600 & 3200 \\
\hline rerr $(f)$ & 0.0199 & 0.0080 & 0.0041 & 0.0046 & 0.0045 & 0.0033 & 0.0013 & 0.0010 \\
\hline
\end{tabular}

Table 2: Relative errors $\operatorname{rerr}(f)$ with $\varepsilon=0.01, k=2, M=100$ and $p=2$ for different $N$.

\begin{tabular}{lcccccccc}
\hline$N$ & 1 & 3 & 4 & 6 & 7 & 8 & 10 & 11 \\
\hline $\operatorname{rerr}(f)$ & 1 & 0.0019 & 0.0022 & 0.0026 & 0.0035 & 0.0044 & 0.0034 & 0.0055 \\
\hline
\end{tabular}

\section{Numerical Example}

From (1.10), we know that

$$
\begin{aligned}
& (K f)(x)=\sum_{n=1}^{\infty} \frac{1-e^{-\sqrt{n^{2}+k^{2}}}}{n^{2}+k^{2}}\left(f, X_{n}\right) X_{n} \\
& =\int_{0}^{\pi} \frac{2}{\pi} \sum_{n=1}^{\infty} \frac{1-e^{-\sqrt{n^{2}+k^{2}}}}{n^{2}+k^{2}} f(s) \sin (n s) \sin (n x) d s=g(x) .
\end{aligned}
$$

We use trapezoid's rule to approach the integral and do an approximate truncation for the series by choosing the sum of the front $M+1$ terms. After considering an equidistant grid $0=x_{1}<\cdots<x_{M+1}=\pi,\left(x_{i}=(i-1) / M \pi, i=1, \ldots, M+1\right)$, we get

$$
\frac{2}{\pi} \sum_{i=1}^{M+1} \sum_{n=1}^{N} \frac{1-e^{-\sqrt{n^{2}+k^{2}}}}{n^{2}+k^{2}} f\left(x_{i}\right) \sin \left(n x_{i}\right) \sin \left(n x_{j}\right) h=g\left(x_{j}\right)
$$

where

$$
h=\frac{\pi}{M}
$$

Example 4.1. It is easy to see that the function $u(x, y)=\left(1-e^{-\sqrt{2} k y}\right) \sin k x$ and the function $f(x)=-2 k^{2} \sin k x$ are the exact solutions of the problem (1.1). Consequently, the data function is $g(x)=\left(1-e^{-\sqrt{2} k}\right) \sin k x$, and

$$
\|f(\cdot)\|_{H^{p}(0, \pi)}=\left(\sum_{n=1}^{\infty}\left(1+n^{2}\right)^{p}\left|f, X_{n}\right|^{2}\right)^{1 / 2}=\left(1+k^{2}\right)^{p / 2} k^{2} \sqrt{2 \pi}
$$

Adding a random distributed perturbation to each data function, we obtain vector $g^{\mathcal{\delta}}$; that is,

$$
g^{\delta}=g+\varepsilon \operatorname{randn}(\operatorname{size}(g)) .
$$

The function "randn $(\cdot)$ " generates arrays of random numbers whose elements are normally distributed with mean 0 , variance $\sigma^{2}=1$, and standard deviation $\sigma=1$. "Randn $(\operatorname{size}(g))^{\prime \prime}$ 
Table 3: $\delta, \alpha$ and $\operatorname{rerr}(f)$ with $p=1 / 2, k=2$ and $E=14.9931$.

\begin{tabular}{lccccc}
\hline$\varepsilon$ & $10^{-1}$ & $10^{-2}$ & $10^{-3}$ & $10^{-4}$ & $10^{-5}$ \\
\hline$\delta$ & 0.0332 & 0.0331 & $3.2100 \times 10^{-4}$ & $3.0044 \times 10^{-5}$ & $2.8271 \times 10^{-6}$ \\
$\alpha$ & 0.0510 & 0.0231 & 0.0109 & 0.0049 & 0.0022 \\
$\operatorname{rerr}(f)$ & 0.1018 & 0.0529 & 0.0607 & 0.0621 & 0.0624 \\
\hline
\end{tabular}

Table 4: $\delta, \alpha$ and $\operatorname{rerr}(f)$ with $p=1, k=2$ and $E=22.4200$.

\begin{tabular}{lccccc}
\hline$\varepsilon$ & 0.1 & 0.05 & 0.01 & 0.005 & 0.001 \\
\hline$\delta$ & 0.0287 & 0.0150 & 0.0029 & 0.0015 & $2.8461 \times 10^{-4}$ \\
$\alpha$ & 0.0067 & 0.0040 & 0.0011 & $6.1518 \times 10^{-4}$ & $1.6699 \times 10^{-4}$ \\
$\operatorname{rerr}(f)$ & 0.0376 & 0.0156 & 0.0035 & 0.0015 & $4.8988 \times 10^{-4}$ \\
\hline
\end{tabular}

returns an array of random entries that is the same size as $g$. The total noise level $\delta$ can be measured in the sense of root mean square error (RMSE) according to

$$
\delta=\left\|g^{\delta}-g\right\|_{2}=\left(\frac{1}{M+1} \sum_{n=1}^{M+1}\left(g_{n}-g_{n}^{\delta}\right)^{2}\right)^{1 / 2}
$$

Using $g^{\delta}$ as data function, we obtain the computed approximation $f_{\alpha}^{\delta}(x)$ by using (3.7). The relative error is given as follows:

$$
\operatorname{rerr}(f):=\frac{\left\|f_{\alpha}^{\mathcal{\delta}}-f\right\|_{2}}{\|f\|_{2}}
$$

where $\|\cdot\|_{2}$ is defined by (4.6).

Tables 1-2 show $M$ and $N$ have small influence on the relative error when they become larger. So, we will always take $M=100$ and $n=7$ in the following examination.

\section{Test 1}

We choose $p=1 / 2, p=1, p=2$ and $p=3$ in Tables $3,4,5$, and 6 to compute the parameters $\delta, \alpha$, and $\operatorname{rerr}(f)$, respectively.

These tables indicate that parameter $\delta, \alpha$, and $\operatorname{rerr}(f)$ all depend on the perturbation $\varepsilon$. $\delta, \alpha$, and $\operatorname{rerr}(f)$ decrease with the decrease of $\varepsilon$. These are consistent with our regularization methods. In addition, $\operatorname{rerr}(f)$ decreases with the increase of $p$ at first, but it ceases decreasing when $p$ reaches to some extent. This means that $\operatorname{rerr}(f)$ does not decrease for stronger "smoothness" assumptions on the exact solution $f(x)$.

\section{Test 2}

Figure 1 shows the comparison between the exact solution $f(x)$ and the regularization solution $f_{\alpha}^{\mathcal{S}}(x)$ for $k=1, k=2, k=3$, and $k=4$ with the perturbation $\varepsilon=0.1, \varepsilon=0.01$, and $\varepsilon=0.001$ with Example 4.1. 
Table 5: $\delta, \alpha$ and $\operatorname{rerr}(f)$ with $p=2, k=2$ and $E=50.1326$.

\begin{tabular}{lccccc}
\hline$\varepsilon$ & $10^{-1}$ & $10^{-2}$ & $10^{-3}$ & $10^{-4}$ & $10^{-5}$ \\
\hline$\delta$ & 0.0300 & 0.0151 & 0.0028 & 0.0014 & $2.8037 \times 10^{-4}$ \\
$\alpha$ & 0.0245 & 0.0174 & 0.0075 & 0.0052 & 0.0024 \\
$\operatorname{rerr}(f)$ & 0.0300 & 0.0105 & 0.0036 & $9.2789 \times 10^{-4}$ & $3.7118 \times 10^{-4}$ \\
\hline
\end{tabular}

Table 6: $\delta, \alpha$, and $\operatorname{rerr}(f)$ with $p=3, k=2$ and $E=112.0998$.

\begin{tabular}{lccccc}
\hline$\varepsilon$ & $10^{-1}$ & $10^{-2}$ & $10^{-3}$ & $10^{-4}$ & $10^{-5}$ \\
\hline$\delta$ & 0.0293 & 0.0133 & 0.0028 & 0.0015 & $2.8145 \times 10^{-4}$ \\
$\alpha$ & 0.0369 & 0.0269 & 0.0144 & 0.0111 & 0.0058 \\
$\operatorname{rerr}(f)$ & 0.0352 & 0.0135 & 0.0051 & 0.0024 & $6.4392 \times 10^{-4}$ \\
\hline
\end{tabular}
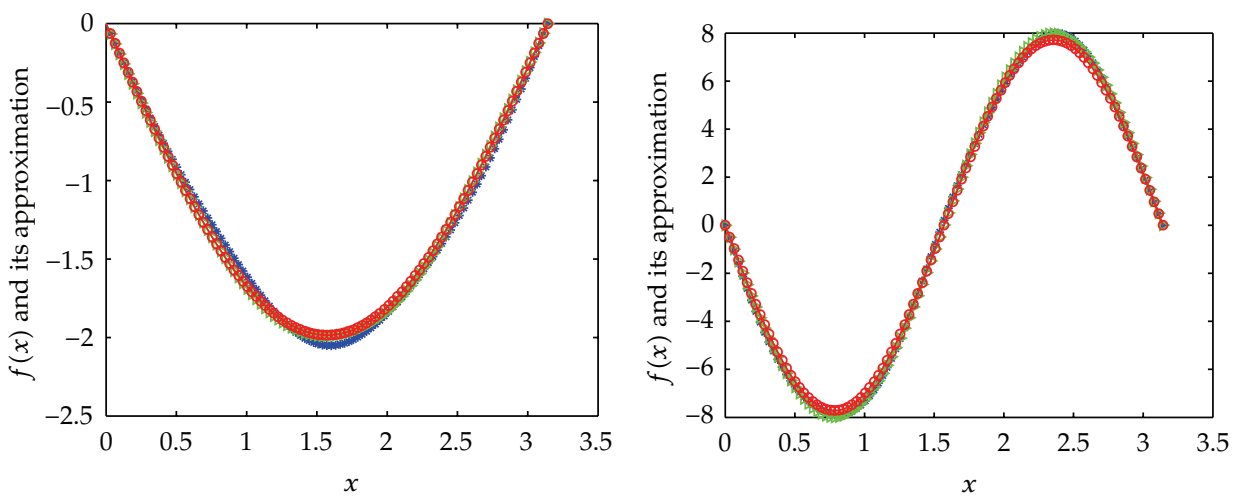

$\begin{array}{ll}-- \text { Exact solution } & \rightarrow \varepsilon=0.01 \\ \rightarrow-\varepsilon=0.1 & \multimap \varepsilon=0.001\end{array}$

(a)

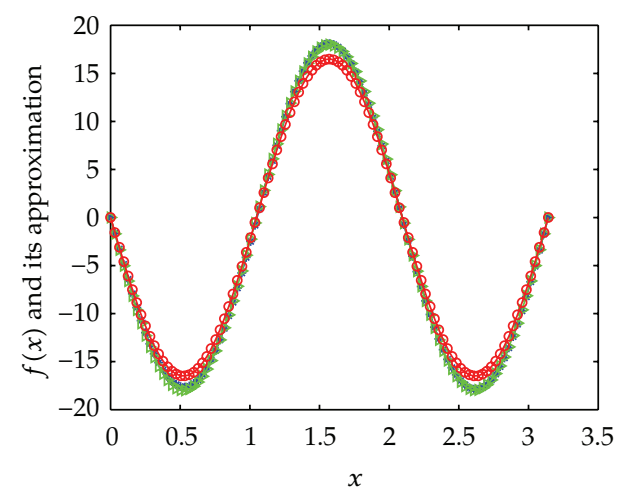

- - Exact solution $\rightarrow \varepsilon=0.01$

* $\varepsilon=0.1$

(c)
- - - Exact solution $\rightarrow \varepsilon=0.01$

(b)

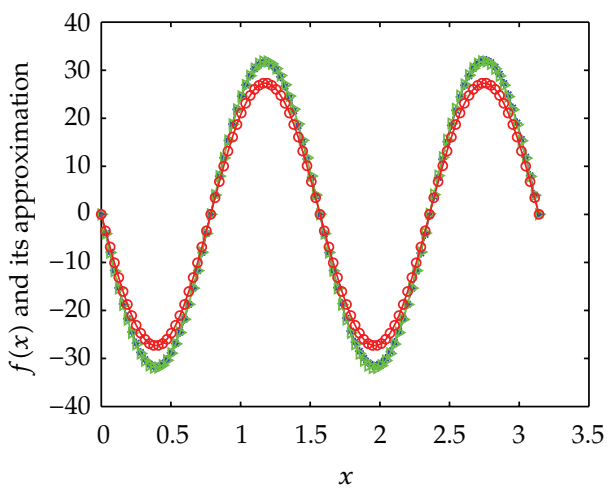

- - Exact solution $\quad \rightarrow \varepsilon=0.01$

$-*-\varepsilon=0.1$

(d)

Figure 1: The exact solution $f(x)$ and its approximation $f_{\alpha}^{\delta}(x)$ : (a) $k=1$, (b) $k=2$, (c) $k=3$, and (d) $k=4$. 


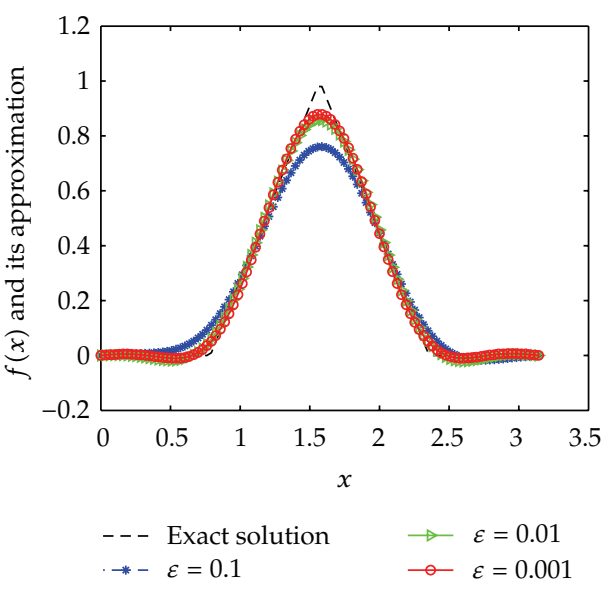

(a)

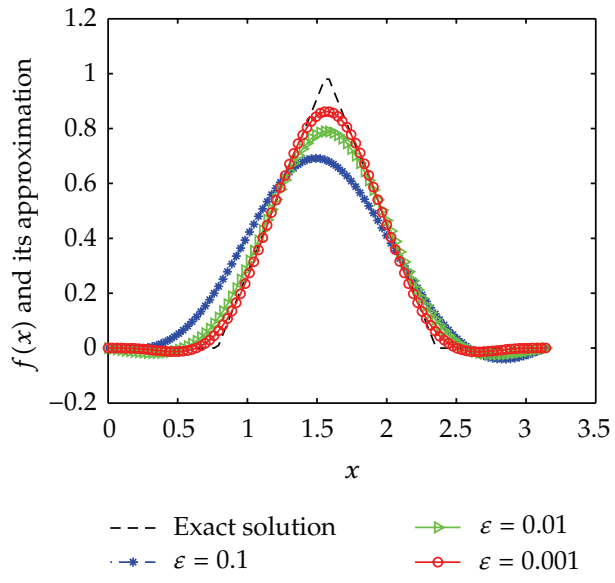

(b)

Figure 2: The exact solution $f(x)$ and its approximation $f_{\alpha}^{\mathcal{\delta}}(x)$ for $k=10$ : (a) $p=2$, and (b) $p=3$.

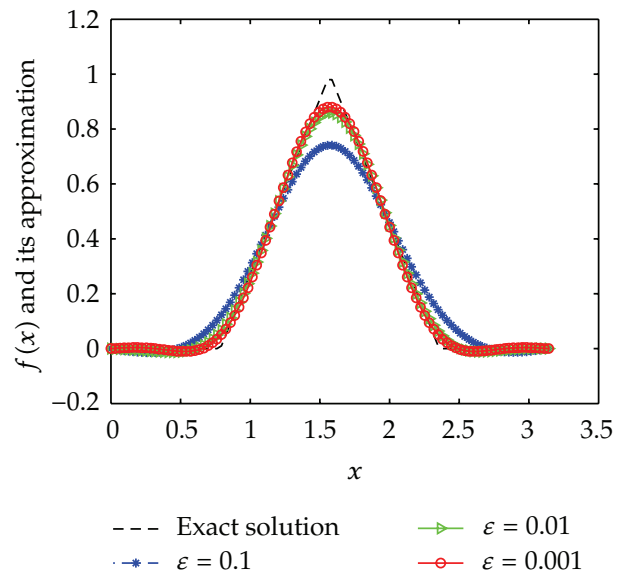

(a)

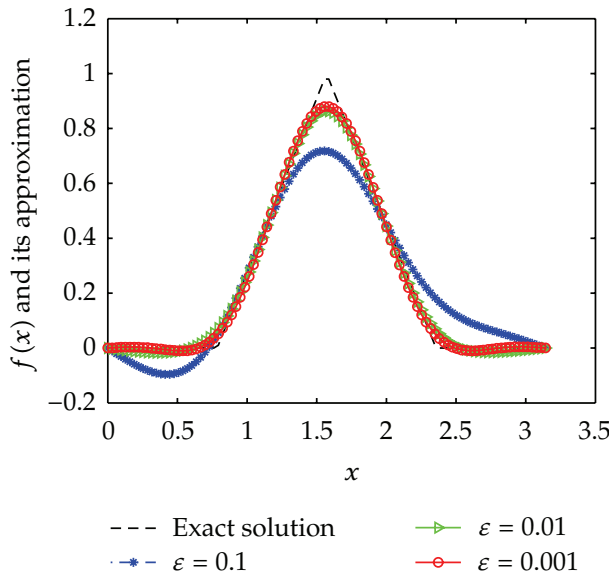

(b)

Figure 3: The exact solution $f(x)$ and its approximation $f_{\alpha}^{\mathcal{\delta}}(x)$ for $p=2$ : (a) $k=3$, and (b) $k=10$.

Figure 1 indicates these regularized solutions approximate to the exact solution, as the amount of $\varepsilon$ decreases, while the numerical results are not so good as the parameter $k$ becomes larger.

\section{Test 3}

The unknown sources $f(x)$ is given. The numerical test was constructed in the following way. First, we selected the solution $f(x)$ and obtained the exact data function $g(x)$ using (1.6). Then, we added a normally distributed perturbation to each data function giving vectors $g_{\delta}(x)$. Finally, we obtained the regularization solutions using (3.7). 
Example 4.2. Consider a piecewise smooth source:

$$
f(x)= \begin{cases}0, & 0 \leq x \leq \frac{\pi}{4} \\ \frac{4}{\pi} x-1, & \frac{\pi}{4}<x \leq \frac{\pi}{2} \\ 3-\frac{4}{\pi} x, & \frac{\pi}{2}<x \leq \frac{3 \pi}{4} \\ 0, & \frac{3 \pi}{4}<x \leq \pi\end{cases}
$$

In Example 4.2, since the direct problem with the heat source $f(x)$ does not have an analytical solution, the data $g(x)$ is obtained by solving the direct problem. Figures 2-3 show the comparisons between the exact solution and its computed approximation with different noise level for Example 4.2. It can be seen that as the amount of noise $\varepsilon$ decreases, the regularized solutions approximate better the exact solution. In addition, even when $k=10$, the regularized solutions still approximate the exact solution. From Figures 2-3, it can be seen that the numerical solution is less than that of Example 4.1. It is not difficult to see that the well-known Gibbs phenomenon and the recovered data near the nonsmooth points are not accurate. Taking into consideration the ill posedness of the problem, the results presented in Figures 2-3 are reasonable.

\section{Conclusions}

In this paper, we considered the inverse problem of determining the unknown source using the simplified Tikhonov regularization method for the modified Helmholtz equation. It was shown that with a certain choice of the parameter, a stability estimate was obtained. Meanwhile, the numerical example verified the efficiency and accuracy of this method.

\section{Acknowledgment}

The project is supported by the Distinguished Young Scholars Fund of Lan Zhou University of Technology (Q201015).

\section{References}

[1] J. R. Cannon and P. DuChateau, "Structural identification of an unknown source term in a heat equation," Inverse Problems, vol. 14, no. 3, pp. 535-551, 1998.

[2] A. Farcas and D. Lesnic, "The boundary-element method for the determination of a heat source dependent on one variable," Journal of Engineering Mathematics, vol. 54, no. 4, pp. 375-388, 2006.

[3] G. S. Li, "Data compatibility and conditional stability for an inverse source problem in the heat equation," Applied Mathematics and Computation, vol. 173, no. 1, pp. 566-581, 2006.

[4] Zh. Yi and D. A. Murio, "Source term identification in 1-D IHCP," Computers \& Mathematics with Applications, vol. 47, no. 12, pp. 1921-1933, 2004.

[5] L. Yan, F. L. Yang, and C. L. Fu, "A meshless method for solving an inverse spacewise-dependent heat source problem," Journal of Computational Physics, vol. 228, no. 1, pp. 123-136, 2009.

[6] L. Yan, C. L. Fu, and F. L. Yang, "The method of fundamental solutions for the inverse heat source problem," Engineering Analysis with Boundary Elements, vol. 32, pp. 216-222, 2008

[7] H. W. Cheng, J. F. Huang, and T. J. Leiterman, "An adaptive fast solver for the modified Helmholtz equation in two dimensions," Journal of Computational Physics, vol. 211, no. 2, pp. 665-674, 2006. 
[8] A. Kirsch, An Introduction to the Mathematical Theory of Inverse Problems, Springer, New York, NY, USA, 1996.

[9] A. Carasso, "Determining surface temperatures from interior observations," SIAM Journal on Applied Mathematics, vol. 42, no. 3, pp. 558-574, 1982.

[10] C. L. Fu, "Simplified Tikhonov and Fourier regularization methods on a general sideways parabolic equation," Journal of Computational and Applied Mathematics, vol. 167, no. 2, pp. 449-463, 2004.

[11] W. Cheng, C. L. Fu, and Z. Qian, "A modified Tikhonov regularization method for a spherically symmetric three-dimensional inverse heat conduction problem," Mathematics and Computers in Simulation, vol. 75, no. 3-4, pp. 97-112, 2007.

[12] W. Cheng, C. L. Fu, and Z. Qian, "Two regularization methods for a spherically symmetric inverse heat conduction problem," Applied Mathematical Modelling, vol. 32, no. 4, pp. 432-442, 2008

[13] C. W. Groestsch, The Theory of Tikhonov Regularization for Fredholm Equation of the First Kind, Longman Sc \& Tech, Boston, Mass, USA, 1984. 


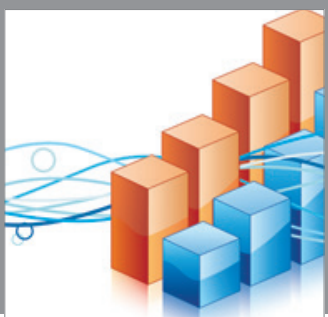

Advances in

Operations Research

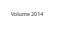

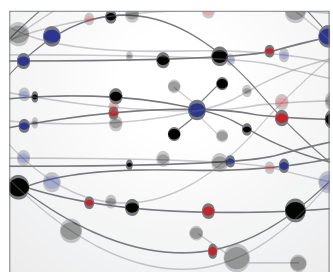

\section{The Scientific} World Journal
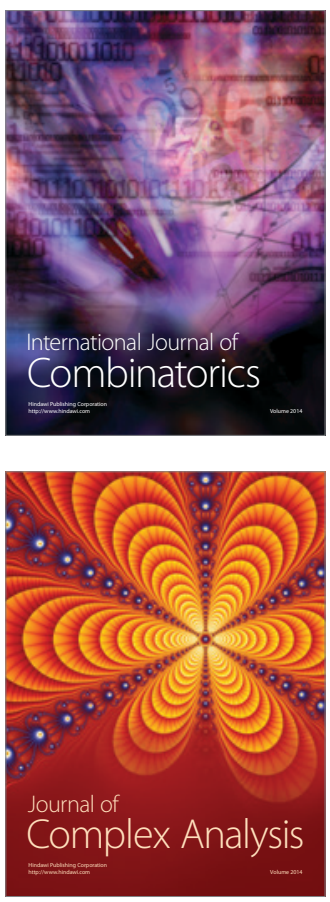

International Journal of

Mathematics and

Mathematical

Sciences
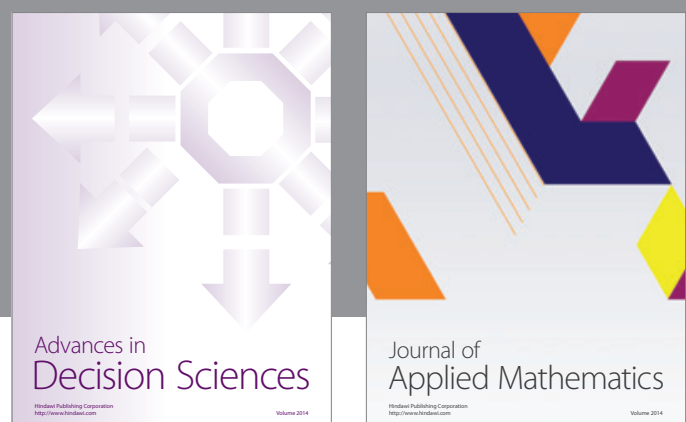

Journal of

Applied Mathematics
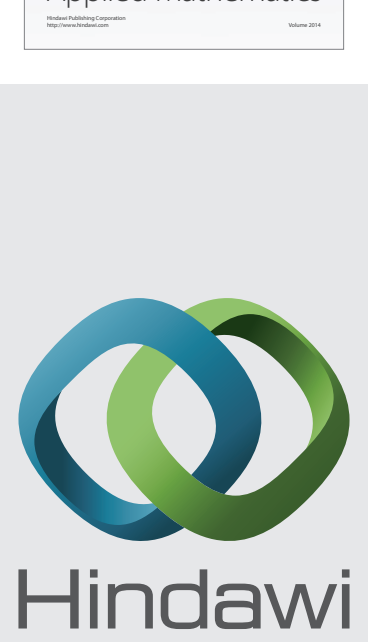

Submit your manuscripts at http://www.hindawi.com
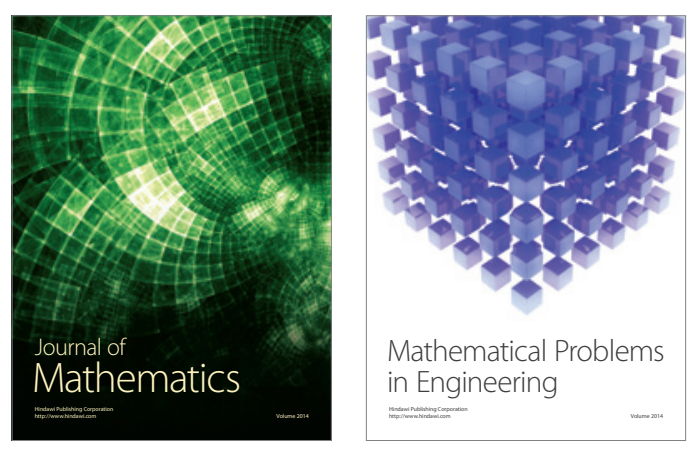

Mathematical Problems in Engineering
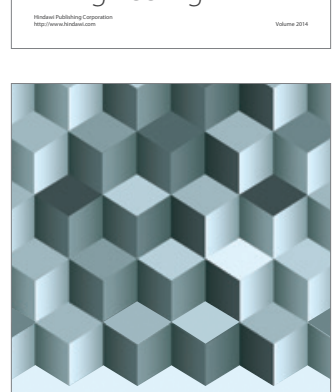

Journal of

Function Spaces
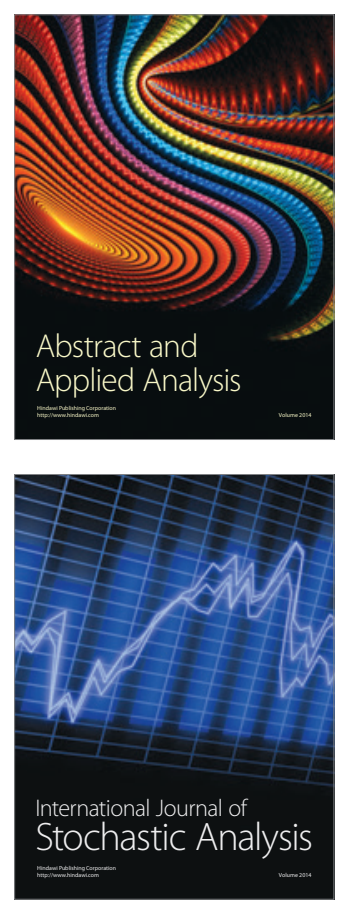

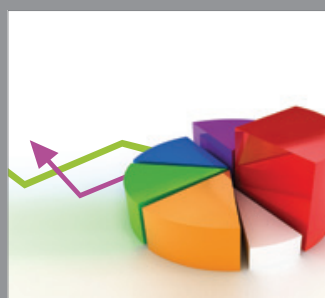

ournal of

Probability and Statistics

Promensencen
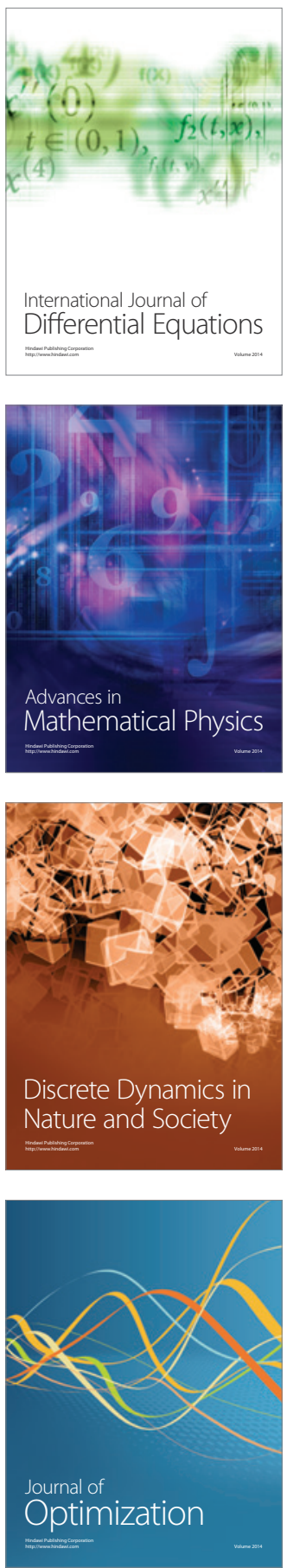